\title{
¿Hacia una casa común? La Comunidad Europea ante las políticas de Gorbachov (1985-1988)
}

\section{Towards a common home? The European Community and Gorbachev's policies (1985-1988)}

\author{
Emanuele Treglia \\ Universidad Complutense de Madrid, España \\ emanutre@ucm.es \\ https://orcid.org/0000-0003-1531-5833 \\ Ricardo Martín de la Guardia \\ Universidad de Valladolid, España \\ guardia@fyl.uva.es \\ https://orcid.org/0000-0003-2595-898X
}

Recibido: 24/09/2021

Aceptado: 08/11/2021

Cómo citar este artículo: TREGLIA, Emanuele; MARTÍN DE LA GUARDIA, Ricardo (2022). ¿Hacia una casa común? La Comunidad Europea ante las políticas de Gorbachov (1985-1988). Pasado y Memoria. Revista de Historia Contemporánea, (24), pp. 91-115, https://doi.org/10.14198/PASADO2022.24.04

\section{Resumen}

Mijaíl Gorbachov, una vez alcanzado el poder en la URSS en 1985, puso en marcha un amplio programa de reformas que aspiraba a democratizar y liberalizar el sistema soviético en múltiples ámbitos, a la vez que apostaba decididamente por mejorar las relaciones con la Comunidad Europea (CE). Al principio los dirigentes de la CE recelaron del «nuevo pensamiento» impulsado por Gorbachov, pensando que su discurso era uno más en la lista de las estrategias propagandísticas del PCUS. Sin embargo, la puesta en práctica de medidas extraordinariamente novedosas, acompañadas de una franca disposición al diálogo y a la negociación en cuestiones clave como la carrera armamentística y los derechos humanos, fueron convenciendo paulatinamente a los líderes comunitarios de la sinceridad de su mensaje. El presente artículo, centrándose

C2022 Emanuele Treglia y Ricardo Martín de la Guardia 
en el período 1985-1988, pretende analizar cómo las instancias comunitarias miraron a las políticas desarrolladas por el nuevo líder soviético y recibieron sus propuestas de acercamiento.

Palabras clave: Comunidad Europea; Unión Soviética; Mijaíl Gorbachov; Perestroika; Casa común europea; Guerra fría; Nuevo pensamiento.

\begin{abstract}
Mikhail Gorbachev, who came to power in the USSR in 1985, launched a wide-ranging reform program aimed, on the one hand, at democratizing and liberalizing the Soviet system in a variety of scenarios and, on the other, at improving relations with the European Community (EC). The EC leaders were initially wary of the «new thinking» promoted by Gorbachev, believing that his discourse was just one more on the list of the CPSU's propaganda strategies. However, the implementation of innovative measures, accompanied by a frank readiness for dialogue and negotiation on key issues such as the arms race and human rights, gradually convinced the EC leaders of the sincerity of Gorbachev's approach. Focusing on the period 1985-1988, this paper aims to analyze how the Community viewed the policies developed by the new Soviet leader and received his proposals for rapprochement.
\end{abstract}

Keywords: European Community; Soviet Union; Mikhail Gorbachev; Perestroika; European Common Home; Cold War; New Thinking.

Financiación: El presente artículo se enmarca en el proyecto La construcción europea desde el Sur. De la ampliación mediterránea a la ampliación al norte (1986-1995): los contornos de la europeización en perspectiva comparada. Financiado por el Ministerio de Ciencia e Innovación. Código: PID2020-113623GB-100. Emanuele Treglia se ha beneficiado también de una Vibeke Sørensen Fellowship (European University Institute-Historical Archives of the European Union).

\title{
Introducción
}

El 11 de marzo de 1985, Mijaíl Gorbachov era designado por el Comité Central del Partido Comunista de la Unión Soviética (PCUS) secretario general, después de una suerte de interregno durante el cual dos ancianos dirigentes -Yuri Andrópov y Konstantín Chernenko- estuvieron al frente de la URSS sin que introdujeran políticas reformistas de las que tan necesitada estaba la superpotencia. El nuevo líder adoptó un talante muy distinto al de sus predecesores, preocupado desde un principio por regenerar la estructura política y económica de un país en declive. Entre 1985 y 1991, Gorbachov puso en marcha un amplio programa de reformas que, anclado en el llamado «nuevo pensamiento» (English, 2000), aspiraba a democratizar y liberalizar el sistema soviético en múltiples ámbitos, a la vez que apostaba decididamente 
por mejorar las relaciones tanto con Estados Unidos, como con la Comunidad Europea (CE). Efectivamente, abandonando la hostilidad hacia el proceso de integración comunitaria que había caracterizado la política exterior de la URSS desde los años cincuenta, el nuevo líder del PCUS impulsó un acercamiento a la CE que superase la división del continente: de ahí sus llamamientos a favor de la construcción de la «casa común» europea.

El presente artículo, centrándose en el período 1985-1988, pretende analizar cómo las instancias comunitarias miraron a las políticas desarrolladas por el nuevo líder soviético y recibieron sus propuestas de acercamiento ${ }^{1}$. Los dirigentes de la CE, la cual se encontraba entonces en un periodo exultante de su historia tras la firma del Acta Única y la ampliación hacia el Sur, recelaron en un principio del «nuevo pensamiento» impulsado por Gorbachov, pensando que su discurso era uno más en la lista de las estrategias propagandísticas del PCUS. No obstante esta inicial desconfianza, la puesta en práctica de medidas extraordinariamente novedosas, acompañadas de una franca disposición al diálogo y a la negociación en cuestiones clave como la carrera armamentística y los derechos humanos, fueron convenciendo paulatinamente a los líderes comunitarios de la sinceridad del mensaje gorbachoviano. Se hizo así posible una aproximación que, en 1988, llevó la CE a normalizar sus relaciones con el Consejo de Ayuda Mutua Económica (CAME), la URSS y otros países del Este. Si bien desde 1989 las políticas de Gorbachov experimentaron una crisis que culminó en 1991 con la disolución de la Unión Soviética, los proyectos analizados en el presente artículo marcaron un antes y un después en la historia más reciente de Europa.

\section{Entre recelos y tímidas esperanzas}

El 13 de marzo de 1985, en la Plaza Roja, tuvo lugar el funeral de Chernenko. Gorbachov, recién elegido secretario general del PCUS, utilizó el entierro de su predecesor como un valioso foro diplomático, celebrando numerosas reuniones con destacados jefes de Estado y de Gobierno europeos que habían acudido a Moscú para la ocasión, como Helmut Kohl -canciller de la República Federal Alemana (RFA)-, Margaret Thatcher -primera ministra del Reino Unido-, François Mitterrand -presidente de la República Francesa- y Sandro Pertini -presidente de la República Italiana-. En estos encuentros, Gorbachov hizo

\footnotetext{
1. Si la formulación de la «casa común» por parte de Gorbachov ha sido objeto de unos notables estudios realizados por Marie-Pierre Rey (2004 y 2008), escasa atención ha sido dedicada a la actitud adoptada por las instituciones comunitarias hacia la perestroika. A este propósito, cabe mencionar el trabajo pionero de Aline Sierp (2015).
} 
hincapié ante sus interlocutores de la necesidad de liberar Europa de las armas nucleares y parar la carrera armamentista, «mostrando una amplia disposición al desarrollo del diálogo» entre los dos bloques². Unos días más tarde, un informe británico sobre los cambios que se estaban dando en la cúspide del Kremlin expresaba al mismo tiempo recelos y un muy tímido optimismo. Por un lado, advertía que no se debía perder de vista el hecho de que Gorbachov había sido «moldeado» por el «sistema soviético»y «educado según los conceptos y el lenguaje del marxismo y el leninismo», no pudiendo ser considerado un «liberal». Por el otro, reconocía que era un «hombre inteligente y propenso a entablar discusiones genuinas» con Europa occidental, lo que abría «la posibilidad de relaciones Este-Oeste más constructivas ${ }^{3}{ }^{3}$.

No solo el Reino Unido, sino el conjunto de los Estados miembros de la CE se mostraba «muy cauto en su valoración del nuevo líder soviético», como hacía notar el Grupo de Trabajo sobre Europa del Este de la Comisión Europea en una reunión celebrada a finales de marzo:

«Muchas delegaciones -se afirmaba en aquella ocasión- piensan que internamente no podemos esperar cambios sustanciales. En sus discursos, Gorbachov refleja en buena medida la línea de Andropov, consistente en implementar la eficiencia (especialmente la económica) sin cambiar la línea política: en otras palabras, no hay nada malo dentro del sistema soviético, que solo necesita funcionar mejor».

Asimismo, el Grupo subrayaba que en la «política exterior» del Kremlin no se podían «identificar aún nuevos objetivos específicos», siendo todavía pronto para saber si las genéricas declaraciones de Gorbachov a favor del diálogo Este-Oeste y del control armamentístico aspiraban realmente a avances significativos o, al contrario, consistían en una maniobra propagandística dirigida, entre otras cosas, a crear división entre EEUU y sus aliados europeos. El hecho de que Andrei Gromyko siguiera siendo ministro de Asuntos Exteriores, un cargo que ocupaba desde 1957, hacía presagiar una sustancial continuidad en este ámbito. En suma, los miembros de la CE temían que Gorbachov, con su talante «informal» que le hacía resultar «más atractivo a la opinión pública» que sus predecesores, imprimiera a las políticas de la URSS «un cambio de estilo, pero no de contenido» ${ }^{4}$.

2. «Visita del Presidente della Repubblica on. Sandro Pertini (Mosca, 12-13 marzo)», Testi e documenti sulla politica estera dell'Italia. 1985, p. 326.

3. The Death of Chernenko and Gorbachev's Succession, 21-III-1985, National Archives, PREM $19 / 1647-2$.

4. USSR. The succession of Mr. Gorbachev, 26-III-1985, Archives Historiques de la Commission Europeenne (AHCE), BAC 250/1980, n. 1409. 
En las semanas siguientes, el líder soviético empezó a dar cierta forma a la que fue definida como una «ofensiva de paz»: propuso la congelación del desarrollo de los arsenales nucleares estratégicos por parte de las dos superpotencias y anunció la moratoria unilateral del despliegue de los misiles SS-20 en Europa oriental. Aunque la nueva estrategia de política exterior moscovita todavía carecía de una formulación detallada y parecía «limitada a un vago conjunto de intenciones», estas iniciativas reflejaban la voluntad de ganar credibilidad e inaugurar un nuevo clima en las relaciones entre el bloque oriental y el occidental (Grachev, 2008: 57). Un paso más en este sentido se dio a finales de mayo, cuando viajó a Moscú Bettino Craxi, primer ministro italiano, que estaba ejerciendo también como presidente de turno del Consejo de la CE. El líder soviético le comunicó que no solo quería «resucitar el espíritu, la atmósfera y la sustancia de la distensión», sino que aspiraba a que se diera un «salto cualitativo». A este propósito, refiriéndose a las conversaciones en curso con EEUU que habrían culminado en noviembre con la celebración de la cumbre de Ginebra, dijo que la URSS estaba dispuesta a negociar una amplia reducción de los armamentos nucleares estratégicos y de alcance medio en Europa a condición de que Washington renunciara a la Iniciativa de Defensa Estratégica (IDE) y su militarización del espacio. La novedad más significativa del encuentro, en todo caso, concernió a la actitud del Kremlin hacia el proyecto de integración de Europa occidental. Gorbachov anunció a Craxi la intención de la URSS y del CAME de establecer relaciones oficiales con Bruselas. Subrayó las ventajas económicas mutuas que podían derivar de este acercamiento y añadió que valoraba positivamente el creciente papel político desempeñado por la Comunidad, a través de los instrumentos de la Cooperación Política Europea (CPE), en el escenario internacional: «En la medida en que los países de la CE actúen como una entidad política, -afirmó el líder del PCUS- estamos dispuestos a buscar con ellos un lenguaje común también sobre problemas internacionales concretos» ${ }^{5}$.

En la sesión plenaria del Parlamento Europeo (PE) del 11 de junio se señaló que los planteamientos expuestos por Gorbachov en su encuentro con Craxi parecían marcar «una ruptura y un giro» con respecto a la anterior posición soviética que «relegaba la Comunidad al rango de un simple instrumento del capitalismo americano». No obstante, para evitar caer en «trampas», se subrayaba la necesidad de evaluar de manera prudente y «realista el contenido de la

5. «Visita del Presidente del Consiglio on. Craxi (Mosca, 28-30 maggio)», Testi e documenti sulla politica estera dell'Italia. 1985, pp. 327-334. 
nueva visión de Europa» que parecía haber adoptado el Kremlin ${ }^{6}$. Tres días más tarde, la Comisión Europea recibió una carta con la que el CAME pedía oficialmente la puesta en marcha de contactos dirigidos a la normalización de sus relaciones con la Comunidad, es decir, al reconocimiento diplomático mutuo, y a la conclusión de acuerdos en varios ámbitos, empezando por el comercial. El desarrollo del diálogo entre las dos entidades resultaba en línea con los principios inspiradores de la Conferencia sobre la Seguridad y Cooperación en Europa (CSCE), orientados a la creación de medidas de confianza entre los dos bloques. Por consiguiente, la Comisión contestó afirmativamente, pero indicó que la CE, si bien estaba dispuesta a abrir negociaciones con el CAME en cuanto institución, a corto plazo quería privilegiar el desarrollo de las relaciones bilaterales con sus distintos Estados miembros, «de manera acorde a sus circunstancias e intereses particulares» ${ }^{7}$. Al ser escéptica hacia el nuevo rumbo gorbachoviano y al constatar las diversas naturalezas de CE y CAME -economía de mercado vs. economía centralizada, organización supranacional vs. organización internacional, etc.-, la Comisión Europea optaba por un enfoque gradualista: prefería ir tanteando el terreno mediante contactos individualizados, a la espera de ver cómo habrían efectivamente evolucionado las políticas del Kremlin y el conjunto del bloque soviético. Además, confiaba en que el desarrollo de la cooperación con Estados como Polonia y Hungría habría contribuido a alimentar las demandas de reformas que iban emergiendo allí cada vez con más fuerza ${ }^{8}$.

Así como la CE esperaba que para los países del Este el incremento de sus relaciones con Bruselas conllevara una gradual reducción de su dependencia política y económica de la URSS, por su parte Moscú buscaba «un mayor contacto con Europa occidental para alejarla de la influencia de Washington» y «acercarla a sus puntos de vista ${ }^{9}$. A este propósito hay que subrayar que, hasta 1987, el enfoque de Gorbachov sobre la cuestión europea fue en gran parte «táctico». Para el líder del PCUS, el diálogo con la CE y sus Estados miembros tenía en sí mismo una importancia limitada, representando sobre todo una herramienta para influir en las relaciones con EEUU. Las primeras referencias a la «casa común europea», aunque contenían ya una cierta pulsión idealista, quedaban todavía vagas y servían sobre todo para que la diplomacia soviética

6. «Sitting of Tuesday, 11 June 1985», Official Journal of the European Communities (OJEC), 2-327, 1985.

7. «Sitting of Wednesday, 23 October 1985», OJEC, 2-331, 1985.

8. Report on relations between the European Community and the countries of Central and Eastern Europe, 7-X-1985, Historical Archives of the European Union (HAEU), PE2-17737.

9. «Comecon courting Western Europe», New York Times, 22-IX-1986. 
ampliara sus interlocutores y, consecuentemente, sus medios de presión en el marco de las negociaciones con Washington sobre el control armamentístico (Rey, 2008: 24-27).

Los contactos de la CE con los países del Este y el CAME -como se verá en las próximas páginas- empezaron a desarrollarse significativamente solo desde la segunda mitad de 1986, conforme se fue configurando un clima de confianza más extenso. Mientras tanto, en el verano de 1985, Gorbachov impulsó importantes cambios en el núcleo dirigente soviético. Ascendieron a posiciones clave algunos de sus colaboradores más cercanos, como por ejemplo el reformista Alexander Yakovlev, al frente del departamento de Propaganda del Comité Central de PCUS; por su parte, Gromyko fue sustituido como ministro de Asuntos Exteriores por Eduard Shevardnadze. Éste, aunque carecía de experiencia diplomática previa, era apreciado por Gorbachov por su «sentido de lo nuevo» y la «originalidad de sus enfoques» ${ }^{10}$. Profundizando en la apuesta por el control armamentístico y la distensión, una de las primeras medidas del tándem Gorbachov-Shevardnadze fue la declaración, en agosto, de una moratoria unilateral de los ensayos nucleares soviéticos. En otoño, la cumbre de Ginebra entre EEUU y la URSS no produjo ningún resultado concreto debido a la persistente desconfianza mutua y a la negativa de Reagan de abandonar la IDE. Aun así, el Consejo Europeo manifestó satisfacción por el relanzamiento del diálogo entre las dos superpotencias, a la vez que expresó «la esperanza de que los nuevos líderes soviéticos» dieran «pruebas concretas de un deseo real de establecer relaciones constructivas entre Este y Oeste». En este sentido, el Consejo precisó: «Ciertos obstáculos todavía se interponen en el camino del regreso a la distensión, incluida la continuación de la ocupación soviética de Afganistán» ${ }^{11}$.

El escepticismo de la Comunidad hacia las políticas de Gorbachov no se debía solo a controversias relativas a la política exterior soviética -como el caso afgano-, sino también al hecho de que el carácter autoritario del modelo vigente en el «socialismo real» seguía sustancialmente inmutable. Así, en diciembre, el presidente de turno del Consejo de la CE, el luxemburgués Jacques Poos, trazaba un balance ambivalente de la labor desempeñada por Gorbachov desde su llegada al frente del PCUS. Por un lado, Poos reconoció que «en las relaciones Este-Oeste el pesimismo característico de los últimos años» había sido «superado, dejando paso a un cierto cauto optimismo»; pero, por el otro, subrayó que no iba a ser «posible un progreso sustancial en las

10. The Diary of Anatoly Chernyaev. 1985, p. 67, https://nsarchive.gwu.edu.

11. «Report from the European Council on European Union (3-XII-1985)», European Political Cooperation Documentation Bulletin (EPCDB), 2:1, 1986. 
áreas de seguridad o cooperación económica» entre las dos Europas mientras los países del bloque soviético siguieran sin garantizar el «respeto firme de los derechos humanos y de las libertades fundamentales» ${ }^{12}$.

Efectivamente, como indicaba con tono "pesimista» un informe redactado por los embajadores de los Doce en Moscú en enero de 1986, Gorbachov no había puesto todavía en marcha ninguna iniciativa relevante dirigida a la aplicación efectiva de los principios contenidos en el tercer cesto de Helsinki ${ }^{13}$, lo que contribuía a la persistencia, entre la opinión pública y las fuerzas políticas occidentales, de importantes recelos acerca de la sinceridad y el alcance del nuevo rumbo soviético. Durante la visita de Gorbachov a Francia en octubre, por ejemplo, varios de sus interlocutores -incluido el primer ministro Laurent Fabius- habían recalcado la cuestión de las violaciones los derechos humanos en la URSS ${ }^{14}$. Asimismo, en el Foro Cultural celebrado en Budapest entre octubre y noviembre bajo el umbral de la CSCE, se había producido un sintomático desencuentro entre los representantes de los países de la CE y los del bloque oriental. Los primeros habían hecho hincapié en el valor de la libertad de la cultura como elemento esencial de una sociedad pluralista y democrática. Los segundos, en cambio, habían defendido la idea de que la producción cultural debía estar al servicio de la colectividad y el Estado socialista, lo que justificaba limitar su libertad cuando se consideraba que podía resultar perjudicial para los intereses de las masas populares. Estas visiones divergentes habían impedido que en el Foro se llegara a la adopción de un documento común, mostrando la distancia que aún separaba las dos Europas ${ }^{15}$.

Ante la desconfianza occidental, en 1986 Gorbachov fue multiplicando sus propuestas. De hecho, ya el 15 de enero presentó un ambicioso plan para llegar, en el año 2.000, a la completa abolición de las armas nucleares a escala internacional. Un mes más tarde se celebró el XXVII Congreso del PCUS. En aquella ocasión, a la vez que trazó las principales líneas de actuación de la perestroika -promoviendo la introducción de elementos liberalizadores en el ámbito económico- y apuntó a la necesidad de proceder a cierta democratización socio-política, Gorbachov avanzó hacia una formulación más definida de lo que fue denominado como el «nuevo pensamiento» en política exterior: abandonó la tradicional visión soviética de las relaciones internacionales basada

12. «Sitting of Wednesday, 11 December 1985», OJEC, 2-333, 1985.

13. Mise en oeuvre par l'Union soviétique des dispositions de l'Acte final d'Helsinki (1er abril 1985-31 décembre 1985), 21-I-1986, AHCE, BAC 250/1980, n. 1413.

14. "Gorbachev's Charm Offensive», The Time, 14-X-1985.

15. Forum de la Culture (Budapest - 15 octobre au 25 novembre 1985), 20-I-1986, AHCE, BAC 250/1980, n. 1413. 
en el principio de la lucha de clases, abrazando definitivamente una postura que enfatizaba la importancia de la cooperación y del entendimiento mutuo para encontrar soluciones compartidas a los desafíos colectivos que afrontaba la humanidad (Dallin, 2004). El desastre de Chernobyl -26 de abril-, al hacer patente el potencial destructivo de una guerra nuclear, reforzó estas orientaciones a favor del diálogo y el desarme y, al mismo tiempo, hizo que se acelerara el desarrollo de la glasnost en el ámbito cultural e informativo (Zubok, 2007: 288-289).

En una reunión celebrada en mayo, el Grupo de Trabajo sobre Europa del Este de la Comisión Europea se preguntó si las iniciativas gorbachovianas, que habían experimentado una verdadera «inflación» en el último período, tenían «solo un objetivo propagandístico»o iban a permitir «alcanzar acuerdos»: «No se llegó a una conclusión clara, ya que algunos sintieron que las propuestas soviéticas deberían primero ser puestas a la prueba de las negociaciones». A propósito de las reformas internas, el Grupo consideraba que estas iban a tener un alcance reducido puesto que Gorbachov no discutía la vigencia del sistema socialista en cuanto tal, sino que se limitaba a hablar de cómo revitalizarlo. Los participantes en la reunión observaron también que la URSS seguía viendo «el dominio» sobre sus satélites como «una prioridad»y, consecuentemente, advirtieron de que probablemente Moscú iba a intentar «controlar las relaciones de los países del Este con la Comunidad». Precisamente por lo que concernía a la CE, el Grupo notaba que para la política soviética la «apertura europea» tenía todavía «poca importancia» de por sí, siendo subordinada a las «relaciones entre las dos superpotencias» ${ }^{16}$.

Aunque a mediados de 1986 en Europa occidental persistían esos importantes recelos hacia el nuevo rumbo del Kremlin, sobre todo en los sectores progresistas iban emergiendo voces esperanzadas que subrayaban la oportunidad de adoptar una actitud más receptiva ante las iniciativas de Moscú. En junio, por ejemplo, un editorial de El País afirmaba que se iba afianzando cada día más «la sensación de que en la diplomacia de Gorbachov» había «aspectos nuevos» que merecían «ser discutidos a fondo»: «Un rechazo sistemático -advertíapodría echar a perder posibilidades quizá históricas ${ }^{17}$.

16. Politique soviétique suite au XXVIIème Congrès du PCUS, 23-V-1986, AHCE, BAC 250/1980, n. 1413. La desconfianza de la opinión pública de Europa Occidental puede verse, por ejemplo, en «Le dynamisme orthodoxe de M. Gorbatchev», Le Monde, 27-II-1986.

17. «Confusión nuclear», El País, 16-VI-1986; «Perché Gorbaciov continua a bussare», La Repubblica, 14-VI-1986. 


\section{Percepciones cambiantes}

A principios de julio se celebró en Moscú un encuentro entre Gorbachov y Mitterrand. Uno de los principales temas tratados fue el de las negociaciones URSS-EEUU sobre el desarme. El líder soviético reiteró su disposición a pactar una notable reducción de los arsenales nucleares, a condición de que los norteamericanos renunciaran a la IDE: «Si dejamos que la carrera armamentística llegue al espacio -dijo-, la situación se volverá ingobernable. [...] Debemos aprender a vivir juntos». Gorbachov buscaba en este sentido el apoyo de Francia y de los otros miembros de la CE, destacando que Europa podía «jugar un papel importante destinado a influir en las conversaciones Este-Oeste». Mitterrand, que con cierto espíritu crítico afirmaba que sobre Reagan pesaba «la influencia del complejo militar-industrial», compartía con el líder soviético la hostilidad hacia la IDE, considerando que esta representaba «un elemento problemático» que podía contribuir a «una escalada». El presidente francés, quien no perdía de vista el hecho de que Gorbachov se había formado en el sistema soviético, seguía manteniendo cautelas. Sin embargo, al mismo tiempo, creía que el líder del PCUS tenía «una inteligencia rápida y flexible», poseía «un agudo sentido de la realidad» y, «sin dejar de defender intransigentemente sus intereses», estaba genuinamente interesado en alcanzar compromisos en varios ámbitos, empezando por el de la limitación de $\operatorname{armamentos}^{18}$. Así, Mitterrand, como otros dirigentes socialistas de Europa occidental -entre ellos Felipe González-, iba paulatinamente desarrollando una percepción positiva del «nuevo pensamiento» gorbachoviano.

Este proceso de cambio de percepción se acentuó a raíz de la cumbre de Reikiavik, celebrada entre la URSS y EEUU en octubre. En aquella ocasión, el líder del PCUS propuso una reducción del 50\% de las armas nucleares estratégicas de ambas superpotencias y la completa eliminación de los misiles de alcance intermedio soviéticos y estadounidenses en Europa. Una vez más, la negativa de Reagan a abandonar la IDE impidió lograr un acuerdo (Hunt y Reynolds, 2016: 160-165). Esta postura norteamericana suscitó notables críticas en Europa occidental. En efecto, el Parlamento Europeo aprobó una resolución en la cual afirmaba:

«[El PE] considera que es totalmente inaceptable que un programa armamentístico como la IDE, que, de ser realizable, beneficiaría exclusivamente a los intereses estadounidenses, se convierta en un impedimento para las medidas

18. Las citas proceden de las transcripciones realizadas por el colaborador de Mitterrand, Jacques Attali (1995a), correspondientes a los días 7-VII-1986 y 31-VII-1986. 
viables en favor del desarme [...] y ve en ello un menosprecio de los intereses específicos de los Estados europeos en materia de seguridad $»^{19}$.

Profundizando en esta línea, el eurodiputado socialista Pieter Dankert observó que, «desde un punto de vista estrictamente objetivo, Gorbachov» había sido «en Reikiavik un mejor aliado para Europa que Reagan»: «Esto puede ser una situación demencial para unos países que para su seguridad dependen de su relación con los Estados Unidos» ${ }^{20}$. En otras resoluciones, el PE solicitaba que se procediera urgentemente a una potenciación de los mecanismos de la CPE y «a la definición de una postura europea» independiente para que los Doce, coordinando sus esfuerzos y hablando con voz unitaria, pudieran hacer valer ante las dos superpotencias los intereses específicos de la $\mathrm{CE}^{21}$. En este marco se iba asomando la idea de que la Comunidad, que estaba experimentando un importante proceso de fortalecimiento y desarrollo institucional -piénsese en la implementación del Acta Única y la ampliación a los países ibéricos-, mediante la puesta en marcha de un renovado diálogo con el bloque soviético podía incrementar su capacidad de incidir significativamente, con un perfil autónomo, en las dinámicas internacionales. Ya en diciembre de 1985 un informe del Ministerio de Asuntos Exteriores italiano había afirmado a este propósito:

«La apertura de Gorbachov hacia la integración europea [...] representa una evolución hacia un reconocimiento más pleno de la realidad política de Europa occidental. [...] La creciente capacidad de los Doce de presentarse en las relaciones con la URSS [...] como un polo unitario [...] puede, si es gestionada con claridad y coherencia, fortalecer su influencia, credibilidad y peso político» ${ }^{22}$.

Retomando planteamientos que habían ya caracterizado experiencias como la de la Ostpolitik, se consideraba que la CE, tanto por su posición geográfica como por sus rasgos históricos y socio-políticos, podía y debía desempeñar un papel democratizador de las relaciones internacionales, configurándose como un puente entre Este y Oeste. En la sesión del PE celebrada el 21 de enero de 1987, por ejemplo, el eurodiputado del PSOE Eusebio Cano Pinto sostuvo que, si se quería evitar que entre el Kremlin y el Pentágono siguiera existiendo

19. «Resolución sobre la necesidad de una auténtica política europea de seguridad tras el fracaso del encuentro de Reikiavik»(23-XI-1986), Diario Oficial de las Comunidades Europeas (DOCE), C-297, 1986.

20. «Sesión del martes 21 de octubre de 1986», DOCE, 2-344, 1986.

21. «Resolución sobre las conversaciones entre Reagan y Gorbachov en Reikiavik» (23XI-1986) y «Resolución sobre los aspectos políticos y económicos de la seguridad en Europa» (23-XI-1986), DOCE, C-297, 1986.

22. Ministero degli Affari Esteri, Rapporti Est-Ovest, 12-XII-1985, https://digitalarchive. wilsoncenter.org/document/155174. 
«un foso terrorífico de guerra fría o caliente, de miedo y de inseguridad», era «necesario que ese foso» fuera «llenado por la presencia de [...] la Europa de los Doce»: «En este sentido, la Europa de los Doce se convertiría en el árbitro y en el amortiguador [...] de la tensión permanente entre las dos superpotencias». Esta perspectiva se veía alentada por los avances registrados en los contactos de la CE con el CAME y sus Estados miembros. Efectivamente, en la misma sesión del PE, Willy De Clercq -comisario europeo de Relaciones Exteriores y de Comercio- señaló que, en la segunda mitad de 1986, la actitud del bloque oriental con respecto a la Comunidad había experimentado una «doble evolución» positiva. Por un lado, todos los países del Este habían comunicado a la Comisión Europea su voluntad de «normalizar las relaciones con la CE» sobre base bilateral, dando los primeros pasos oficiales hacia la apertura de negociaciones. Por el otro, el CAME, que como institución había ido entablando sus propias conversaciones con la CE -su secretario Vyacheslav Sychev se había entrevistado con De Clercq en septiembre-, había reconocido finalmente «la competencia individual de los países de Europa del Este» a la hora de desarrollar relaciones con Bruselas ${ }^{23}$. El hecho de que se hubiera concedido este margen de autonomía a los Estados del «socialismo real» alimentaba la credibilidad del «nuevo pensamiento» soviético. Asimismo, en ambientes comunitarios se cultivaba la esperanza de que estos múltiples contactos con el CAME y sus miembros -sobre los cuales se volverá en las próximas páginasconstituyeran «el punto de partida de la reconciliación entre las dos partes de Europa», contribuyendo a la gradual superación de Yalta ${ }^{24}$.

Desde finales de 1986 empezó a presentarse bajo una nueva luz también la situación de los derechos humanos en la URSS. En noviembre, con ocasión de la apertura de la reunión de Viena de la CSCE, Jean-Bernard Raimond y Hans-Dietrich Genscher -ministros de Asuntos Exteriores de Francia y Alemania Occidental-argumentaron en un artículo conjunto que, si se quería que la «casa común europea» fuera realmente «habitable», hacía falta garantizar en ella el respeto de las libertades fundamentales ${ }^{25}$. Un mes más tarde, la emblemática liberación del disidente y Premio Nobel de la Paz Andréi Sájarov demostró que Gorbachov estaba comenzando a actuar significativamente en ese sentido, introduciendo medidas dirigidas a aliviar los rasgos autoritarios y los mecanismos represivos del sistema soviético (Snyder, 2011: 174 y ss.).

23. «Sesión del miércoles 21 de enero de 1987», DOCE, 2-347, 1987.

24. Rapport sur les relations entre la Communauté européenne et le CAEM et les Etats membres du CAEM, 19-XII-1986, HAEU, PE2-18043. Véase también Maslen (1986).

25. Hans-Dietrich Genscher y Jean-Bernard Raimond, «Rendre la maison européenne habitable», Le Monde, 5-XI-1986. 
Así, reunidos en el marco de la CPE, el 19 de diciembre los representantes de los Doce hacían notar con satisfacción que recientemente en la URSS habían «sido liberados algunos ciudadanos inocentes cuyo único 'crimen' consistía en creer en el derecho de libertad de opinión». Detectaban también «alguna mejora, aunque limitada, en la manera en la que los medios soviéticos» reflejaban «los problemas de la sociedad». Asimismo, valoraban positivamente el hecho de que se permitieran con más facilidad las reunificaciones familiares entre ciudadanos de los dos lados del telón de acero -especialmente de las dos Alemanias-. En todo caso, los Doce lamentaban la persistencia de muchas «sombras», subrayando que, a pesar de esos avances, el ejercicio de las libertades de asociación, de movimiento, de creencia y de expresión seguía sufriendo demasiadas restricciones ${ }^{26}$.

En 1987, la percepción del «nuevo pensamiento» soviético por parte de la opinión pública y de las fuerzas políticas de Europa occidental continuó mejorando, conforme Gorbachov lo fue dotando de mayor profundidad y concreción. A finales de enero, por ejemplo, en un pleno del Comité Central del PCUS el líder del Kremlin enfatizó la necesidad de proceder a una seria democratización de las dinámicas y estructuras políticas de la URSS, empezando por el propio partido: propuso, entre otras cosas, que los secretarios del PCUS a todos los niveles fueran designados por los respectivos comités mediante elecciones que contaran con distintas candidaturas y escrutinio secreto (Brown, 1996: 166-167). El Financial Times afirmó que medidas de este tipo, aunque no eran precisamente «lo que Occidente» entendía «como democracia»-dado que no ofrecían alternativas al PCUS en cuanto tal-, podían «ser un significativo punto de partida para hacer menos monolítico el control del Partido Comunista sobre el país» y también «menos monopolístico» ${ }^{27}$. Cuando el 28 de febrero Gorbachov se dijo dispuesto a negociar la eliminación de los misiles de alcance intermedio en Europa sin que Washington renunciara a la IDE, el mismo periódico escribió que, si bien no se podía descartar que detrás de esa propuesta hubiera «segundas intenciones», era ya «hora de respaldar» al líder soviético en este ámbito ${ }^{28}$. En marzo, a raíz de un viaje a la URSS durante el cual tuvo «discusiones francas» en una «atmósfera amistosa», la propia primera ministra británica, Margaret Thatcher, hasta entonces escéptica hacia el nuevo rumbo soviético, reconoció que las reformas puestas en marcha por Gorbachov, aunque iban «a necesitar tiempo para ser efectivas», representaban

26. «Statement at the CSCE Vienna Follow-up Meeting (19-XII-1986)», EPCDB, 2:2, 1986.

27. «One cheer for Mr. Gorbachev», Financial Times, 28-I-1987.

28. «Time to back Mr. Gorbachev», Financial Times, 2-III-1987. 
indudablemente «un punto de inflexión» ${ }^{29}$. También el Gobierno español, según comunicó Felipe González al líder del PCUS un par de meses más tarde, valoraba «positivamente los esfuerzos» hechos por las dos superpotencias «en los foros de desarme» y recibía «con interés y esperanza las noticias sobre el proceso de transformaciones» que se estaba desarrollando en la URSS ${ }^{30}$.

Como se decía en un informe redactado en marzo por Emile Noël -secretario de la Comisión Europea-, en el último período había aumentado la fascinación por Gorbachov incluso en un país tradicionalmente hostil a la URSS como la República Federal Alemana: resultaba «destacable» que no solo las izquierdas, sino también los sectores conservadores y de derechas se pronunciaban «por lo menos a favor de un examen atento» de las reformas internas y de la política exterior del Kremlin ${ }^{31}$. El eurodiputado de la Unión Demócrata Cristiana (CDU) Hans-Gert Pöttering, por ejemplo, afirmó en el PE que era «preciso congratularse» con Gorbachov por «abrir la política soviética hacia dentro y hacia fuera», añadiendo: «En interés de las personas de la Unión Soviética [...], pero también en interés de la paz en Europa y en el mundo, debemos desear éxito a Gorbachov en su política». Esto, de todas formas, no significaba que los recelos de los conservadores de la RFA hacia el nuevo rumbo gorbachoviano hubieran ya desaparecido. El mismo Pöttering, de hecho, remarcaba las sombras que seguían oscureciendo las actuaciones del Kremlin, como la perpetuación de «la guerra soviética contra el pueblo afgano» ${ }^{32}$. Asimismo, el canciller democristiano Helmut Kohl decía sentir por Gorbachov una «simpatía escéptica», considerando que el líder del PCUS, lejos de querer construir una «república democrática», aspiraba a establecer un comunismo más eficiente, lo que podía resultar peligroso para Occidente porque habría incrementado el «poder de seducción soviético» (Rey, 2004: 51).

Mientras tanto, la percepción de Europa Occidental por parte del Kremlin fue experimentando una significativa evolución. Desde la primavera, en efecto, el diálogo con la CE dejó de abordarse por Moscú a través de aquel enfoque táctico que hasta entonces le había atribuido un papel primordialmente instrumental en el marco de las relaciones URSS-EEUU. La colaboración con Bruselas empezó a ser considerada como un asunto importante per se. En este sentido,

29. Meeting of the Cabinet, 2-IV-1987, National Archives, CAB 128-85-13. Sobre el viaje véase Brown (2020: 195-200).

30. Carta de F. González a M. Gorbachov, 14-V-1987, Archivo de la Fundación Felipe González (AFFG), Correspondencia, 53.08.13.

31. Rapports et commentaires dans la presse allemande sur les réformes et les propositions de M. Gorbatschow, 14-III-1987, HAEU, EN-1005.

32. «Sesión del jueves 12 de marzo de 1987», DOCE, 2-350, 1987. 
Gorbachov declaró a los miembros del Politburó que la Comunidad debía pasar a ocupar una posición clave en la política exterior soviética, convirtiéndose en un interlocutor privilegiado del Kremlin, porque sin ella no era posible «decidir nada importante» (Rey, 2008: 28). En el otoño, estos planteamientos llevaban los Doce a constatar que, «en un grado cada vez mayor», la URSS percibía «la cooperación política europea como una entidad relevante y una fuerza en la política internacional $»^{33}$. Asimismo, Gorbachov fue dotando de mayor profundidad y carga idealista la formulación de la "casa común europea», subrayando que Europa occidental y oriental compartían no solo intereses estratégicos y geopolíticos, sino también raíces históricas y culturales. De esta manera, el líder soviético apuntaba a una idea de «integridad» que sobrepasaba el hecho de que los Estados europeos tuvieran diferentes sistemas socio-políticos: «La casa es común, -escribió en su libro Perestroika, publicado después del verano[...] pero cada familia tiene su propio apartamento [...]. Mas solo trabajando juntos $[\ldots]$ los europeos pueden salvar su casa, [...] mejorarla y hacerla más segura» (1987: 195). El desarme, la colaboración económica, los intercambios culturales y el respeto de la llamada dimensión humana eran algunos de los pilares fundamentales de esa «casa común» que, en la visión gorbachoviana, constituía una pieza cardinal de una nueva arquitectura internacional superadora de la Guerra Fría.

En este clima de creciente confianza mutua, en octubre, una delegación del Soviet Supremo de la URSS realizó por primera vez una visita oficial al Parlamento Europeo. El viaje fue promovido por el Grupo Socialista que desde 1985 estaba trabajando activamente para favorecer el acercamiento entre Bruselas y Moscú (Athanasopoulou, 2020). La delegación se reunió con casi todos los grupos del PE -la única excepción fue representada por la extrema derecha-, lo que evidenció que en la CE se había afianzado un amplio consenso acerca de la oportunidad de impulsar el reencuentro entre las dos Europas. Durante su visita, los soviéticos trataron numerosos temas. Entre otras cosas, hablaron de derechos humanos y reiteraron tanto su apuesta por la glasnost y la perestroika, como su voluntad de llegar a corto plazo a una considerable reducción de los armamentos nucleares y convencionales. Quizás la novedad más significativa consistió en el hecho de que dieron luz verde a la inclusión de la llamada «cláusula territorial»-el reconocimiento de que los tratados internacionales firmados por la Comunidad se aplicaban al territorio de Berlín Oeste- en la declaración común que estaba siendo negociada por la CE y el CAME. Dado que el rechazo del Kremlin a dicha cláusula se había ido

33. «Report of the European Council on European Union (24-XI-1987)», EPCDB, 3:2, 1987. 
convirtiendo en el principal obstáculo que impedía a las dos entidades alcanzar un acuerdo, la nueva postura soviética allanaba el camino para que -como escribía proféticamente el presidente del Grupo Socialista Rudi Arndt-en 1988 se llegara finalmente al establecimiento de «relaciones diplomáticas oficiales no solo entre el CAME y la CE, sino también entre [sus] distintos Estados miembros» $»^{34}$.

\section{Amueblar la «casa común»}

En enero de 1988, en su discurso ante el PE con motivo del inicio de la Presidencia alemana del Consejo de la CE, Hans-Dietrich Genscher -ministro de Asuntos Exteriores de la RFA- afirmó que, gracias al «proceso de transformación» emprendido por la Unión Soviética bajo el liderazgo de Gorbachov, en los últimos años las relaciones Este-Oeste habían experimentado un notable «cambio favorable». Mencionó en este sentido la cumbre EEUU-URSS celebrada en Washington a finales de 1987 -en la cual las dos superpotencias habían firmado el Tratado sobre Fuerzas Nucleares de Rango Intermedio-, describiéndola como un acontecimiento de importancia histórica que había constituido un «paso decisivo hacia un desarme real». Genscher señaló que estaba surgiendo «una nueva era en las relaciones internacionales» e hizo hincapié en la necesidad de que los Doce influyeran significativamente en su «conformación»:

«Para ello -dijo-son necesarios objetivos que marquen la vía hacia el futuro, una identidad inconfundible y la voluntad de dar forma al destino de Europa. [... En una época de dinamización [...] y de cambios de largo alcance en la política mundial, urge progresar en [...] la dimensión de la 'política de seguridad' de la integración europea».

Genscher consideraba que la Comunidad, a la vez que se iba fortaleciendo «en el plano interno», debía dotarse de «una mayor capacidad de acción hacia el exterior», potenciando los mecanismos de la CPE con el fin de que desembocara a corto-medio plazo en la adopción de «una política exterior común». Según el ministro alemán, solamente moviéndose en esta dirección la CE habría podido aprovechar plenamente la ventana de oportunidades abierta por las transformaciones en curso en el bloque soviético, incrementando su propio

34. Report on the Visit of a Delegation from the Supreme Soviet to the European Parliament. Brussels/Strasbourg, 6-13 October 1987, HAEU, GSPE-077. 
estatus en el escenario internacional y contribuyendo al mismo tiempo a la superación de «la división de Europa» ${ }^{35}$.

También en enero de 1988, hablando en calidad de representante de los Doce en el marco de la CSCE, Genscher se refirió a la «casa común». Después de definirla como una formulación «visionaria» que propiciaba «la configuración de un futuro común para todos los europeos», precisó significativamente: «La identidad europea [...] ha encontrado su expresión más consistente en el estado de derecho de la democracia pluralista que hoy es la norma en los Estados de la CE. [...] La forma adoptada por la 'casa común europea' [...] requiere un cierto acuerdo sobre el mobiliario ${ }^{36}$. En la misma línea, unos meses más tarde Jacques Delors - presidente de la Comisión Europea- afirmó:

«La Comunidad debe mantenerse fiel a su modelo basado en el pluralismo político y en el respeto absoluto de los derechos humanos, así como a la economía mixta, en la que el papel del mercado se combina con el de los organismos públicos para lograr un crecimiento económico con concertación social. Estos principios deben sentar las bases de la 'casa común' europea ${ }^{37}$.

En definitiva, según los líderes de la CE el reencuentro de las dos Europas habría debido producirse en el terreno de la democracia liberal. El desarrollo de las reformas promovidas por el Kremlin sugería que Gorbachov estaba paulatinamente abrazando esta perspectiva. De hecho, la retirada de las tropas soviéticas de Afganistán, anunciado oficialmente en abril e iniciado en mayo, acentuó considerablemente la credibilidad del «nuevo pensamiento» en política exterior ${ }^{38}$. En ámbito interno, un acontecimiento clave tuvo lugar en la XIX Conferencia del PCUS, celebrada a finales de junio. Las decisiones adoptadas en aquella ocasión, que aspiraban a poner las bases de un «Estado socialista de derecho», introdujeron en el sistema soviético elementos sustanciales de pluralismo y democracia. Se estableció, por ejemplo, la creación de un nuevo órgano legislativo, el Congreso de los Diputados del Pueblo, cuya composición iba a ser determinada mayoritariamente mediante elecciones competitivas -id est, con múltiples candidatos- que se realizarían por primera vez en 1989 (Brown, 1996:175-184). Como escribió en su diario Anatoly Chernyaev-asesor de política exterior de Gorbachov-, los planteamientos de la XIX Conferencia

35. «Sesión del miércoles 20 de enero de 1988», DOCE, 2-360, 1988. Sobre la necesidad de fortalecer la política exterior de la CE, véase también: «Resolución sobre la Cumbre de Washington» (17-XII-1987), DOCE, C-33, 1988.

36. «Statement at the Opening of the Fifth Session of the Vienna CSCE Follow-up Meeting» (22-I-1988), EPCDB, 4:1, 1988.

37. Summary Report of the Socialist Group EC-COMECON Conference on 2-3 July 1988, HAEU, GSPE-078.

38. «Statement Concerning Afghanistan»(14-IV-1988), EPCDB, 4:1, 1988. 
aspiraban a crear «garantías contra la dictadura, no sólo del individuo sino también del partido», y a «cortar por fin el cordón umbilical» con el burocratismo y con «toda la herencia del estalinismo» ${ }^{39}$.

Mientras en la URSS y en varios países de Europa del Este se iban produciendo cambios que los Doce acogían «con satisfacción $»^{40}$, el 25 de junio la CE y el CAME llegaron a la firma de la tan esperada Declaración Conjunta. Las dos entidades establecían relaciones oficiales e instituían un marco en el cual llevar a cabo futuras conversaciones acerca de modalidades y ámbitos concretos de cooperación ${ }^{41}$. En Pravda, Sychev-secretario del CAME- caracterizó el acuerdo como «un ladrillo más en la construcción de nuestra casa común europea» (Van Oudenaren, 1991: 290). Por su parte, el miembro de la Comisión Europea Willy De Clercq resaltó en El País que «la firma de la declaración» tenía «ante todo un significado político»: «[Es] nuestra contribución a la distensión. También tiene un alcance económico, pero creo que se trata de una inversión a medio plazo ${ }^{42}$. Asimismo, el liberal italiano Enzo Bettiza -nombrado presidente de la recién creada Delegación del PE para las relaciones con la URSS-afirmó que la Comunidad había logrado «tres cosas esenciales»:

«En primer lugar, el reconocimiento [...] también ideológico de esta Europa [...] de la libertad. [...] En segundo lugar, el importantísimo reconocimiento [...] de que el territorio de Berlín [Oeste] es territorio comunitario. En tercer lugar, [...] el principio del bilateralismo. [...] Es importante que cada país del Este europeo, de la Europa central, sepa que puede tener y encontrar en la Comunidad un interlocutor dispuesto a entablar relaciones, negociaciones, a firmar acuerdos de índole comercial a nivel bilateral, de Bruselas a Budapest, de Bruselas a Sofía, de Bruselas a Varsovia, etc.» ${ }^{43}$.

En marzo de 1988, tres meses antes de la firma de la Declaración, Gorbachov explicitaba en una reunión con Alessandro Natta, el líder de los comunistas italianos, su visión de los asuntos europeos a la vez que aportaba algunas claves al respecto. La evolución de Europa occidental desde 1945, y muy especialmente el proceso de integración, era de sumo interés para el Kremlin y, en general, para el mundo socialista. El modelo era distinto del que se promovía desde

39. The Diary of Anatoly Chernyaev. 1988, pp. 19 y 26, https://nsarchive.gwu.edu.

40. «Statement Concerning East-West Relations» (13-VI-1988), EPCDB, 4:1, 1988.

41. «Declaración conjunta sobre el establecimiento de relaciones oficiales entre la Comunidad Económica Europea y el Consejo de Ayuda Mutua Económica», (24-VI1988), DOCE, L-57, 1988.

42. «Entrevista a Willy De Clercq», El País, 9-VI-1988.

43. «Sesión del jueves 16 de junio de 1988», DOCE, 2-366, 1988; Opinión de la Comisión de Relaciones Económicas Exteriores a propósito de la Declaración conjunta sobre el establecimiento de relaciones oficiales entre la CEE y el CAEM, 15-VI-1988, HAEU, PE2-18725. 
Moscú, pero había que contar con él en la Europa de finales de los ochenta; es más, podía ser un ámbito de negociación y cooperación para estrechar relaciones entre los dos sistemas de organización política y económica que compartían el espacio del Viejo Continente. En aquel encuentro Gorbachov dijo: «No observamos el proceso de integración en Europa occidental desde un punto de vista meramente crítico, sino en todos sus aspectos. Nuestros temores derivan solo de la intención de militarizar el proceso $»^{44}$.

En efecto, décadas después de los combativos documentos contra el Mercado común (las Tesis de 1957 y 1962), los miembros del Instituto de Economía Mundial y Relaciones Internacionales de la Academia de Ciencias de la URSS consideraban que la Comunidad Europea ya no se percibía como un rival irreconciliable, sino como un aliado natural en el ámbito europeo; incluso concluían que la CE era la «institución espejo» del CAME y así los expresaron en las Catorce Tesis sobre el Mercado Común, que dejaban obsoletas las anteriores. No era de extrañar, por tanto, que la consecuencia lógica fuera la citada Declaración conjunta aprobada el 25 de junio de $1988^{45}$.

Dicha Declaración allanó el camino para que, de manera acorde al principio del bilateralismo, la Comunidad fuera estableciendo relaciones oficiales con los distintos Estados miembros del CAME. Así, ya en agosto y septiembre la CE firmó acuerdos de reconocimiento diplomático mutuo con la URSS, Hungría, Bulgaria, Alemania Oriental y Polonia. Contemporáneamente, llevó a cabo negociaciones de acuerdos comerciales y de cooperación económica, los primeros de los cuales fueron suscritos con Hungría y Checoslovaquia en septiembre y diciembre ${ }^{46}$.

Precisamente en vista de la normalización de las relaciones diplomáticas, en julio la Comisión de Asuntos Políticos del PE presentó un extenso informe acerca de la situación socio-política de la URSS. En el análisis se reconocían los esfuerzos por la modernización y apertura de la economía soviética, se apreciaba un mayor respeto de los derechos humanos y se valoraban positivamente los avances registrados en la senda de la democratización. Al mismo tiempo, se advertía de que la URSS había entrado en «un período difícil de transición caracterizado por la existencia simultánea de numerosos elementos del sistema antiguo con un número creciente de elementos nuevos». En este sentido, el informe llamaba la atención sobre el hecho de que el afianzamiento de las reformas se presentaba como un proceso largo y complejo, amenazado por

44. Cit. en Neumann (1989: 93).

45. Martín de la Guardia y Pérez Sánchez (2017: 76).

46. Las negociaciones con la URSS, en cambio, no se concluyeron hasta noviembre de 1989

(Smith, 2004; Van Oudenaren, 1991: 282). 
múltiples obstáculos. A las pulsiones nacionalistas centrífugas y a las problemáticas condiciones económicas, se sumaban inercias sociales y resistencias a los cambios por parte de las élites del PCUS. A este propósito el informe afirmaba:

«La perestroika pretende llevar a cabo una transformación controlada en la Unión Soviética. Su éxito depende pues de que el aparato del partido y las administraciones locales y regionales asuman la idea de la reforma a todos los niveles. [...] La mayor parte de la población se mantiene más bien a la expectativa. La perestroika requiere en primer término un reajuste de esquemas mentales $[\ldots]$ y un largo proceso de aprendizaje ${ }^{47}$.

Así, a la vez que iban dejando sustancialmente atrás la desconfianza hacia el «nuevo pensamiento» soviético, a partir de la segunda mitad de 1988 las instancias comunitarias empezaban a mostrar cierta preocupación por un eventual naufragio de los proyectos gorbachovianos. En septiembre, por ejemplo, el Grupo Socialista en el PE declaraba estar «a favor del camino de las reformas», aunque fueran «reformas graduales y parciales», porque cualquier «camino alternativo» no habría conducido «a aquella 'caída del imperio' auspiciada por algunos ultraderechistas, sino a una represión sangrienta y, posiblemente, al advenimiento» de otras «dictaduras policiales o militares» ${ }^{48}$. Dos meses más tarde, constatando que la URSS se encontraba en una situación económica «muy preocupante» y se enfrentaba a «enormes desafíos», el comisario De Clercq sostenía que la CE tenía «todo el interés» en «alentar» y «ayudar»a Gorbachov «en su política de reformas» porque los logros de la perestroika se habrían repercutido beneficiosamente sobre el conjunto de las dinámicas europeas, mientras que su fracaso habría contrarrestado el proceso de distensión en marcha en el continente ${ }^{49}$.

Mientras tanto, en el otoño el líder soviético celebró importantes encuentros con destacados dirigentes de países comunitarios. A finales de octubre, por ejemplo, viajó a Moscú Helmuth Kohl. A su vuelta, escribió a Felipe González que él y Gorbachov habían tenido conversaciones caracterizadas por «realismo y optimismo» en las cuales, por lo que concernía a las relaciones URSS-RFA y URSS-CE, habían manifestado la común intención de «crear confianza paso a paso y desarrollar un amplio diálogo y cooperación a todos los niveles», empezando por el ámbito económico y el de la seguridad ${ }^{50}$. Gorbachov, por

47. Informe elaborado en nombre de la Comisión de Asuntos Políticos sobre las relaciones políticas de la Comunidad Europea con la Unión Soviética, 18-VII-1988, HAEU, PE2-18731.

48. The European Parliament's Socialist Group and current developments in the Soviet Union and Eastern Europe, 1-IX-1988, HAEU, GSPE-078.

49. Intervention du commissaire De Clercq sur l'état des relations avec l'URSS, 16-XI-1988, HAEU, PE2-228.

50. Carta de H. Kohl a F. González, 4-XI-1988, AFFG, Correspondencia, 53.12.05. 
lo tanto, había conseguido «romper el hielo» con el canciller alemán, quien había sido hasta entonces el líder comunitario más escéptico hacia el nuevo rumbo soviético, lo que resultaba emblemático de los avances producidos en la aproximación entre las dos Europas ${ }^{51}$. En noviembre viajó a Moscú también Mitterrand, quien había ya dado muestra de tener una buena sintonía con el líder soviético. En aquella ocasión, el presidente francés dijo a Gorbachov: «Usted habla, a propósito de Europa, de 'casa común'. Es una expresión bonita. [...] El objetivo verdadero es toda Europa. [...] Comparto este objetivo, sin ocultar las dificultades ${ }^{52}$. En estas palabras se pueden notar los gérmenes de un proyecto que Mitterrand habría lanzado a finales de 1989, y para cuyo desarrollo veía en Gorbachov un aliado esencial: el de una confederación europea concebida como un amplio marco institucional en el cual habrían ido gradualmente tomando cuerpo formas de asociación entre los Doce y los países de Europa del Este. Aunque el designio del presidente francés no llegó a concretizarse -las razones de su fracaso exceden el propósito del presente artículo-, su propia formulación era reveladora de las extraordinarias oportunidades que parecían abrirse a escala continental gracias a las transformaciones desencadenadas por el «nuevo pensamiento» soviético ${ }^{53}$.

En el proceso de configuración de una «casa común» fundamentada en el principio de la libertad de elección, un paso decisivo fue representado por el abandono de la llamada «doctrina Brézhnev» que, después de haber empezado a producirse de facto desde finales de 1987, fue anunciado explícita y oficialmente a la comunidad internacional por Gorbachov el 7 de diciembre de 1988, en un discurso ante la Asamblea General de las Naciones Unidas. De esta manera, los países de Europa del Este ya no veían limitada su soberanía por parte del Kremlin, aunque el líder soviético cultivaba la esperanza -que fue revelándose ilusoria a partir de mediados de 1989- de que acabaran decidiendo mantener voluntariamente una relación privilegiada con la URSS (Grachev, 2008: 121-122). En el mismo discurso en la ONU, que fue acogido con «satisfacción» por el $\mathrm{PE}^{54}$, Gorbachov proclamó su intención de efectuar una considerable reducción armamentística unilateral. Además, en la sesión de clausura de la reunión de Viena de la CSCE celebrada en enero de 1989,

51. «Gorbachev Hails Kohl in Moscow», The New York Times, 25-X-1988.

52. Transcripción realizada por Attali (1995b), correspondiente al 25 de noviembre de 1988.

53. Sobre el proyecto de confederación europea, las razones de su fracaso y los contactos entre Mitterrand y Gorbachov en el marco de su desarrollo, véanse Bozo (2008) y Newton (2013).

54. «Resolución sobre las declaraciones del Sr. Gorbachov ante las Naciones Unidas» (15XII-1988), DOCE, C-12, 1989. 
la URSS abrazó considerables compromisos a favor de los derechos humanos (Snyder, 2011: 214-216) lo que supuso, según afirmaron los Doce reunidos en el marco de la CPE, «un hito histórico en el camino hacia una Europa más libre, más segura y más próspera» ${ }^{55}$.

Así, como escribía Felipe González, al empezar 1989 se reconocía en los ambientes comunitarios que los «esfuerzos» de Gorbachov "por realizar amplias reformas en su país y por ayudar a crear un clima de confianza» en las relaciones Este-Oeste habían "contribuido de manera decisiva» a un «mejor conocimiento, entendimiento y cooperación entre los distintos países europeos», redundando en beneficio de «la superación de la división del Continente» e inaugurando la época «más positiva de cuantas ha conocido la vida internacional desde $1945 »^{56}$. Sin embargo, paradójicamente, a partir de entonces la perestroika fue experimentando una creciente crisis que culminó con la disolución de la URSS en diciembre de 1991, imposibilitando consecuentemente la efectiva materialización de la «casa común» según las modalidades imaginadas por Gorbachov.

\section{Conclusiones}

Entre 1985 y principios de 1989 Gorbachov no desaprovechó la ocasión para dar carta de naturaleza a los postulados contenidos en su «nuevo pensamiento». Así ocurrió, por ejemplo, en diciembre de 1988, con motivo de su discurso ante la Asamblea General de la ONU, en donde se comprometió a evitar toda injerencia en los asuntos internos de los países de la Europa del Este. El concepto de política exterior desarrollado por Gorbachov transformaba los fundamentos de la acción exterior soviética con el fin de avanzar en el diálogo con los países occidentales para mejorar las relaciones en todos los ámbitos y desactivar los focos de mayor tensión entre ambos bloques. Indudablemente, el «nuevo pensamiento» constituía un pilar esencial de la perestroika cuya meta era dinamizar la estructura económica gracias a mecanismos novedosos que mejorasen su eficacia. Este cambio de perspectiva trastocó la vida institucional del país. En 1985, apenas llegado al poder, como se ha comentado, Gorbachov reemplazó al sempiterno Gromiko por Shevardnadze en la cartera de Exteriores y este último estaría llamado a ser una figura esencial en la nueva estrategia exterior del país. Tanto el XXVII Congreso del PCUS, que se celebró en Moscú del 25 de febrero al 6 de marzo de 1986, como la XIX Conferencia del mismo,

55. «Statement at the Closing Session of the CSCE Vienna Follow-up Meeting» (18-I-1989), EPCDB, 5:1, 1989.

56. Carta de F. González a M. Gorbachov, 22-II-1989, AFFG, Correspondencia, 53.08.13. 
desarrollada en junio de 1988, recogieron los originales principios del «nuevo pensamiento», sobre todo la concepción de un mundo interdependiente donde la política exterior soviética debía orientarse hacia posiciones pacifistas y avanzar por la senda del entendimiento con los países occidentales.

Sobre estos principios se fraguaron los tres ejes de actuación principales de la URSS de Gorbachov en este terreno: la drástica reducción de la inversión en armamento con el fin de derivar recursos a la mejora de los sectores productivos; el fin de la Doctrina de Soberanía Limitada, que liberaba a los países del Este del rígido sometimiento a las pautas del Kremlin y, por último, un cambio en la política dirigida hacia el Tercer Mundo. Según Gorbachov, había que acomodarse a la situación internacional de finales de la década de los ochenta en donde la tensión entre bloques bien pudiera ser sustituida por el acuerdo para poder afrontar con éxito los retos suscitados por la carrera nuclear y los problemas medioambientales. De hecho, y a pesar de las primeras reticencias, personalidades políticas de la talla de Margaret Thatcher, François Mitterrand, Helmut Kohl y Felipe González, como se ha tenido ocasión de ver, reconocieron la excelente disposición del líder soviético para alcanzar acuerdos de envergadura.

Sin embargo, el entusiasmo que comenzó a provocar Gorbachov en los países de la CE en la segunda mitad de la década de los ochenta corrió paralelo con el descrédito progresivo en su país. Gorbachov había apostado por la viabilidad de un novedoso modelo de socialismo en libertad, pero la evolución de los acontecimientos demostró que el modelo de Partido-Estado soviético no admitía regeneración posible. Si una parte importante de la población veía frustradas sus esperanzas de caminar rápidamente hacia una democracia real y reclamaba cambios más profundos, el desarrollo de las reformas chocaba con la dura resistencia promovida por los sectores ortodoxos del PCUS. Estos y otros factores -entre los cuales cabe destacar las pulsiones nacionalistas centrifugas- hicieron que, desde 1989, los proyectos reformistas de Gorbachov experimentaron una crisis que llevó al definitivo colapso de la URSS en diciembre de 1991.

A partir de 1989, tampoco el devenir político de Europa central y oriental siguió precisamente la senda trazada por Gorbachov, cuya meta era alumbrar una federación sobre la base de una suerte de humanismo socialista. Efectivamente, una vez levantada la «doctrina Brézhnev», los países del Este prefirieron distanciarse del Kremlin y orientarse decididamente hacia Occidente. En los años posteriores, si bien las ampliaciones de la Unión Europea (UE) produjeron el «retorno a Europa» de varios Estados que había formado parte del bloque soviético, las problemáticas relaciones entre Bruselas 
y Moscú hicieron desvanecer la esperanza de construir una «casa común» en la cual la nueva Rusia ocupara una habitación.

\section{Bibliografía}

ATHANASOPOULOU, Alexandra (2020). Mediating in the Cold War: How the Socialist Group of MEPs Became a Driver of Brussels-Moscow Rapprochement. En BROAD, Matthew y KANSIKAS, Suvi (eds.). European Integration Beyond Brussels. Cham: Palgrave-Springer Nature, 71-92.

ATTALI, Jacques (1995a). Verbatim II. 1986-1988. París: Fayard.

ATTALI, Jacques (1995b). Verbatim III. 1988-1991. París: Fayard.

BOZO, Frédéric (2008). The Failure of a Grand Design: Mitterrand's European Confederation, 1989-1991. Contemporary European History, 17, 391-412.

BROWN, Archie (1996). The Gorbachev Factor. Oxford: Oxford University Press. BROWN, Archie (2020). The Human Factor. Nueva York-Oxford: Oxford University Press.

DALLIN, Alexander (2004). The Rise of New Thinking on Soviet Foreign Policy. En BROWN, Archie (ed.). The Demise of Marxism-Leninism in Russia. BasingstokeNueva York: Palgrave Macmillan, 178-191.

ENGLISH, Robert (2000). Russia and the Idea of the West. Nueva York: Columbia University Press.

GORBACHEV, Mikhail (1987). Perestroika. Nueva York: Harper \& Row.

GRACHEV, Andrei (2008). Gorbachev's Gamble. Cambridge: Polity Press.

HUNT, Jonathan y REYNOLDS, David (2016). Geneva, Reykjavik, Washington, and Moscow, 1985-8. En SPOHR, Kristina y REYNOLDS, David (eds.). Transcending the Cold War. Oxford: Oxford University Press, 151-179.

MARTÍN DE LA GUARDIA, Ricardo y PÉREZ SÁNCHEZ, Guillermo Á. (2017). La Unión Soviética ante el espejo de las Comunidades Europeas. De la Europa sovietizada a de la «casa común» europea. Valladolid: Universidad de Valladolid. MASLEN, John (1986). The European Community's Relations with the StateTrading Countries of Europe, 1984-1986. Yearbook of European Law, 6:1, 335-356.

NEUMANN, Iver (1989). Soviet Perceptions of the European Community, 1950-1988. Oslo: Norsk Utenrikspolitisk Institutt.

NEWTON, Julie (2013). Gorbachev, Mitterrand, and the Emergence of the PostCold War Order in Europe. Europe-Asia Studies, 65:2, 290-320.

REY, Marie-Pierre (2004). 'Europe is our Common Home': A study of Gorbachev's diplomatic concept. Cold War History, 4:2, 33-65.

REY, Marie-Pierre (2008). Gorbachev's New Thinking and Europe, 1985-1989. En BOZO, Frédéric et al. (eds.). Europe and the End of the Cold War. Londres-Nueva York: Routledge, 23-35. 
SIERP, Aline (2015). Democratic Change in Central and Eastern Europe 1989-90. Bruselas: European Parliament.

SMITH, Karen (2004). The Making of EU Foreign Policy. Basingstoke-Nueva York: Palgrave Macmillan.

SNYDER, Sarah (2011). Human Rights Activism and the End of the Cold War. Nueva York: Cambridge University Press.

VAN OUDENAREN, John (1991). Detente in Europe. Durham-Londres: Duke University Press.

ZUBOK, Vladislav (2007). A Failed Empire. Chapel Hill: The University of North Carolina Press. 